Disclosure of Interests: None declared

DOI: 10.1136/annrheumdis-2019-eular.4804

\section{FRI0529 SPREAD OF SENESCENCE AND JOINT INFLAMMATION VIA CONNEXIN43-POSITIVE EXOSOMES RELEASED BY OSTEOARTHRITIC CHONDROCYTES}

Marta Varela-Eirín ${ }^{1}$, Adrián Varela-Vázquez ${ }^{1}$, Amanda Guitián-Caamaño ${ }^{1}$, Susana B. Bravo-López ${ }^{2}$, Carlos Paíno ${ }^{3}$, Raquel Largo ${ }^{4}$, Eduardo Fonseca ${ }^{1}$, Mustapha Kandouz ${ }^{5}$, Trond Aasen ${ }^{6}$, Arantxa Tabernero ${ }^{7}$, Alfonso Blanco ${ }^{8}$, José R. Caeiro ${ }^{9}$, María D. Mayán ${ }^{1}$. Instituto de Investigación Biomédica de A Coruña (INIBIC), CellCOM research group, A Coruña, Spain; ${ }^{2}$ Instituto de Investigación Sanitaria de Santiago de Compostela (IDIS), CHUS, USC, Proteomics laboratory, Santiago de Compostela, Spain; " "Ramón y Cajal" Hospital (IRYCIS), Unit of Experimental Neurology-Neurobiology, Madrid, Spain; ${ }^{4}$ IIS-Fundación Jiménez Díaz UAM, Bone and Joint Research Unit, Rheumatology Department, Madrid, Spain; ${ }^{5}$ School of Medicine, Wayne State University, Department of Pathology, Detroit, United States of America; ${ }^{6}$ Vall d'Hebron Research Institute (VHIR), Universitat Autònoma de Barcelona, Translational Molecular Pathology research group, Barcelona, Spain; ${ }^{7}$ Instituto de Neurociencias de Castilla y León (INCYL), Universidad de Salamanca, Departamento de Bioquímica y Biología Molecular, Salamanca, Spain; ${ }^{8}$ UCD Conway Institute of Biomolecular and Biomedical Research, Flow Cytometry Core Technologies, Dublin, Ireland; ${ }^{9}$ Complexo Hospitalario Universitario de Santiago de Compostela (CHUS). Universidade de Santiago de Compostela (USC), Department of Orthopaedic Surgery and Traumatology, Santiago de Compostela, Spain

Background: Chondrocytes $(\mathrm{CHs})$ in articular cartilage undergo phenotypic changes and senescence, restricting cartilage regeneration and favoring osteoarthritis $(\mathrm{OA})$ progression. $\mathrm{CH}$ and synovial cells from $\mathrm{OA}$ patients show a chronic increase in the channel protein connexin43 (Cx43), which regulates signal transduction. Extracellular vesicles (EVs), including exosomes, have been shown to play important roles in many biological functions and harbour $\mathrm{Cx}$ channels that allow the formation of gap junctions (GJs) between the exosome and the target cell, but the role of these EVs and exosomal-Cx43 in OA progression has not been studied yet.

Objectives: The objective of this study was to investigate the role of EVs released by $\mathrm{OA}$ chondrocytes (OACs) in cellular plasticity, inflammation and senescence of surrounding joint tissues.

Methods: $\mathrm{CHs}$, bone and synovial cells were isolated from healthy and OA donors. EVs were obtained by ultracentrifugation and their protein content was analysed by LC-MS/MS. Protein levels were evaluated by western blot, immunofluorescence and flow cytometry. RNA expression was evaluated by RT-qPCR. Senescence and GJ intercellular communication was studied by flow cytometry and scrape loading assay, respectively.

Results: OACs showed increased levels of Cx43 within their EVs in comparison to the EVs isolated from healthy donors. Overexpression of Cx43 in $\mathrm{CHs}$ increased senescence and exosomal Cx43 levels. Interestingly, the treatment of $\mathrm{CHs}$, bone cells and synoviocytes (target cells) with $\mathrm{Cx} 43-\mathrm{EV} s$ released by OACs, led to a significant increase in both Cx43 mRNA and protein levels in the recipient cells. The increase of $\mathrm{C} \times 43$ in target cells acted as a positive regulator of the reversion to a less differentiated state via EMT by activation of Twist-1, associated with increased levels of the mesenchymal markers, as CD105/CD166. The phenotypic changes detected in OACs lead to a decrease in Col2A1 and aggrecan expression in $\mathrm{CHs}$, and increased the levels of cellular senescence and the senescence associated secretory phenotype (SASP) in the target cells in target cells via p53/p16 and NF-kß. These results were corroborated by analysing the protein cargo of these Cx43 positive EVs by LC-MS/MS, finding enrichment in proteins related with catabolic, senescence and wound-healing pathways. Conclusion: Our results indicate that Cx43-positive exosomes released by OACs may be involved in the spread of cellular senescence and inflammation involved in wound healing failure. Further understanding of the role of exosomal $\mathrm{Cx} 43$ in $\mathrm{OA}$ will help to halt the disease spread and progression. Disclosure of Interests: : None declared DOI: 10.1136/annrheumdis-2019-eular.4692

\section{FRI0530 \\ ADIPOSE DERIVED STEM CELLS TRANSPLANTATION AMELIORATES OSTEOARTHRITIS THROUGH AUTOPHAGY INDUCING VIA PI3K/AKT/MTOR SIGNALING}

Fan Lian ${ }^{1}$, Hanjiang Zeng ${ }^{1}$, Keng Chen ${ }^{2}$, Yiming Liu ${ }^{1}$, Yu Wang ${ }^{1} .{ }^{1}$ the first affiliated hospital of Sun Yat-sen University, Guangzhou, China; ${ }^{2}$ the eighth affiliated hospital of Sun Yat-sen University, Guangzhou, China

Background: Osteoarthritis is characterized by joint inflammation and cartilage degradation. Adipose derived stem cells (ADSCs), as a source of adult mesenchymal stem cells, display similar multiple-linage differentiating potentials to bone marrow MSCs, only are much more abundant, much easier to isolate and expand, have been suggested for suppressing inflammatory responses and repairing cartilage damage. Autophagy are known to be take part in the pathogenesis of OA. Aging related changes of chondrocytes were related to increased mammalian target of rapamycin (mTOR) signaling and defective autophagy (1). We aim to determine the effect of ADSC on autophagy and its underlying mechanism.

Objectives: To investigate the effect of ADSCs on autophagy in a rat osteoarthritis model and IL-1-induced chondrocytes, and to ascertain whether it regulates autophagy via PI3K/AKT/mTOR signaling

Methods: ADSCs and chondrocytes were isolated from SD rats. Flow cytometry was performed for ADSC phenotypic characterization. ADSCs and chondrocytes coculture were established in the presence of $\mathrm{IL}-1 \beta$. A rat anterior cruciate ligament transection (ACLT) OA model were established. ADSCs or irrelevant cell lines of similar number were injected in the osteoarthritisaffected joints. Autophagic activation was determined by Western blotting for LC3-II, p62, MDC (monodansylcadaverine) staining and GFP-LC3 fluorescence microscopy. Autophagy inhibition was mediated by siRNA knockdown of ATG5. Relevant proteins in the $\mathrm{PI3K} / \mathrm{AKT} / \mathrm{mTOR}$ signaling pathway were detected by western blotting. IL-1 $\beta$, IL-6, TNF $\alpha$, IFN- $\gamma$, Col2, MMP-1, 3 and -13 were measured. Histology of the target joints were evaluated.

Results: Application of ADSCs resulted in downregulation of MMP13 and upregulation of Col2 in OA model cartilage and IL-1 $\beta$ induced chondrocytes. ADMSCs decreased pro-inflammatory cytokines IL-1 $1 \beta$, IL-6, and TNF- $\alpha$ in rat OA and IL-1 $\beta$-induced chondrocytes. Autophagy was inhibited in OA model and IL-1 $\beta$ induced chondrocytes. ADSCs increased cell viability and autophagy-related proteins levels in vitro and in vivo. In IL $1 \beta$ induced chondrocytes with ATG5 knockdown by siRNA, the effect of ADSCs on autophagy activation and its function as suppression of IL-1 $\beta$ induced pro-inflammatory cytokines was undermined. Furthermore, ADSCs remarkably decreased the expressions of phosphorylated (p)-PI3K, p-AKT and $\mathrm{p}-\mathrm{mTOR}$ in $\mathrm{IL}-1 \beta$ induced chondrocytes.

Conclusion: ADSCs inhibited the progression of cartilage degeneration in a rat $O A$ model and provide an effective approach to decrease the proinflammatory cytokines secretion. ADSCs' anti-inflammatory effect was associated with cell autophagy and these roles of ADSCs may be associated with $\mathrm{PI} 3 \mathrm{~K} / \mathrm{AKT} / \mathrm{mTOR}$ signaling pathway.

\section{REFERENCES}

[1] Alvarez-Garcia O, Olmer M, Akagi R, Akasaki Y, Fisch KM, Shen T, Su Al, Lotz MK. Suppression of REDD1 in osteoarthritis cartilage, a novel mechanism for dysregulated mTORsignaling and defective autophagy.Osteoarthritis Cartilage. 2016; 24(9):1639-47

Disclosure of Interests: None declared

DOI: 10.1136/annrheumdis-2019-eular.718

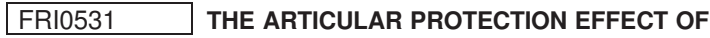 ISORHAMNETIN IN THE RATS OF MONOSODIUM IODOACETATE-INDUCED OSTEOARTHRITIS}

Deng-Ho Yang ${ }^{1}$, Hsiang-Cheng Chen ${ }^{2}$. $T$ Taichung Armed-Forces General Hospital, Taichung, Taiwan, Republic of China; ${ }^{2}$ Tri-Service General Hospital, National Defense Medical Center, Division of Rheumatology/Immunology/Allergy, Department of Internal Medicine, Taipei, Taiwan, Republic of China

Background: Osteoarthritis $(\mathrm{OA})$ is a degenerative joint disease with damage to the articular cartilage. Active production of inflammatory cytokine chemokine and matrix metalloproteinases may be found during the progression of OA. Isorhamnetin had the effects of anti-inflammatory, antioxidant, anti-ischemia, anti-atherosclerotic hepatoprotective and anticancer activities.

Objectives: Our study was focused on the effects of isorhamnetin treatment in $\mathrm{OA}$

Methods: We used monosodium iodoacetate (MIA)-induced $O A$ rats to evaluate the effects of isorhamnetin related anti-inflammatory process. The rats in all groups were sacrificed on four weeks post-MIA injection. The measurements of knee joint swelling, histological analysis, serum inflammatory biomarkers and Western blot were evaluated.

Results: We found that isorhamnetin may reduce MIA-induced knee swelling by significantly reduction of articular cartilage damage in rats. The severity of $O A$ lesion was graded using the modified Mankin scoring sys tem, and we found that the overall modified Mankin's scores were significantly decreased after isorhamnetin treatment compared with a MIA treated group. The production of IL-1 $\beta, \mathrm{IL}-6$, and TNF- $\alpha$ decreased in isorhamnetin treatment group compared with MIA alone group. 